\title{
Energy Aware Scheduling Algorithm for Vehicle Networking Applications in Cloud Computing Platform
}

\author{
Zheng Xiao and Bo Wang \\ Department of Computer Science and Technology, Chengdu Neusoft University, Chengdu 611844, China
}

\begin{abstract}
This paper expounds the strategic significance of China car networking industry development, the development of a comprehensive analysis of the current situation of domestic and foreign car networking industry, the bottleneck problem of all aspects from the development process of different dimensions for China car networking industry in policy, technology, standards and business models. Finally, the cloud platform model, vehicle networking task set model and energy consumption model were proposed, with the energy saving objective function and variable factors determined. Cloud platform environment simulation experiment by simulating the multidimensional resources concurrent tasks, to physical activity time and the number of active server, cloud platform of energy consumption as the performance index, the task set integration algorithm and the existing algorithms are compared. Experimental results show that the TSC algorithm can avoid task set resources in the case of conflict, so that the cloud platform for the application of the cloud platform to activate the number of physical servers to achieve the least, the minimum energy consumption.
\end{abstract}

Keywords-TSC algorithm; car networking; cloud platform; energy consumption model

\section{CAR NETWORKING OVERVIEW}

Car networking for China to build a powerful car and a harmonious society are significant, which as follows:

Firstly, the car networking has a leading role in China's strategic emerging industries. The first car networking is an important part of the Internet of things. As early as 2009, the country has been listed as a strategic emerging industry, which is one of the key areas of traffic promotion demonstration area of one of the 1 . Therefore, the development of the car networking will effectively promote and lead the development of the Internet of things and related strategic emerging industries [1]. Secondly, in 2015 the Prime Minister Li Keqiang in the government work report clearly to put forward the Internet + Action plan to promote the transformation of traditional industries of the Internet [2]. As one of the national pillar industry, the automobile industry in the civilian industry in the most complex, the various aspects of the products and the Technology and Employment Association, driven by strong 2, should become an important carrier of "Internet +" and the effective starting point, and car networking is built "key industries the Internet + car"[3]. Finally, the car networking for the country's transportation industry, information industry, as well as information security is also of great significance. In 2014 Chinese car ownership has reached 154 million units, and in the continued growth in vehicle networking, full vehicle and can effectively gather a large number of users' data, provide support for the construction of intelligent transportation system and information industry upgrade; and in all things Internet era, information security is very important [4]., so the establishment of car networking industry in mainland the enterprise as the main body, will become the premise of ensuring national industrial and traffic information security field 3[5].

\section{SYSTEM MODEL}

This section describes the proposed system model, including the cloud platform model, vehicle networking task set model and energy consumption model.

\section{A. Cloud Platform Model}

Cloud platform model contains a car networking task set integration controller and many of the physical server. In this paper, the cloud platform model reference [6]. The task set integration controller is used to integrate the task set, so that the physical server resources are occupied as little as possible.

Define 1, CloudPlat contains $\mathrm{M}$ server physical server, cloud platform as set CloudPlat $=\left\{\mathrm{Pm}_{1}, \mathrm{Pm}_{2}, \cdots, \mathrm{Pm}_{\mathrm{m}}\right\}$, $\mathrm{Pm}_{\mathrm{i}}$ represents any one physical server in the cloud platform.

The model of cloud platform in each physical server "Pm" _i are isomorphic, all have the same physical resource allocation.

Define 2, a physical server resource with a four dimensional vector include $\left(r_{1}, r_{2}, r_{3}, r_{4}\right)$ to represent. $r_{1}$, represents the number of CPU), $r_{2}$ is for the size of the Memory, $r_{3}$ is for the size of the Disk, $r_{4}$ is for the size of the Network.

Each physical server has a certain number of physical resources, each physical server in order to meet the requirements of the task set, provide resources to the virtual machine.

\section{B. Car Networking Tasks Set Model}

Before explaining car networking tasks set model for the concept of network model of the car. As shown in figure I, this paper car networking model contains three substances, respectively is the information processing center, road test stations, car. Information processing center and the road test station for information exchange, information processing center and to obtain information between vehicle and road test station monitor the operation of the car. 
Resource requirement definition of the 3 car networking task set in a four-dimensional vector $\left(\mathrm{vr}_{1}, \mathrm{vr}_{2}, \mathrm{vr}_{3}, \mathrm{vr}_{4}\right)$ to express. The number of $\mathrm{vr}_{1}$ on behalf of vCPU, the car networking task set the number of CPU required; $\mathrm{vr}_{2}$ represents the size of the vMemory, the car networking task set to Memory $\mathrm{vr}_{3}$ on behalf of vDiks size; the size of the car networking task set required Storage size; ${v r_{4}}_{4}$ represents the size of the Network[7], namely car networking the task sets the demand for Network.

There are many different types of tasks in vehicle networking applications on the cloud platform, task integration according to its own characteristics, composition of task set, then the integration of the car networking task set virtual cluster resource allocation, different task set virtual cluster using physical servers with a cloud platform sharing.

Define 4 vehicle networking tasks set at a particular moment on the requirements of a particular type of resource $\left(\mathrm{T}_{1_{\mathrm{r} 1}}, \mathrm{~T}_{2_{\mathrm{r} 2}}, \mathrm{~T}_{3_{\mathrm{r} 3}}, \mathrm{~T}_{4_{\mathrm{r} 4}}\right)$ to represent. $\mathrm{T}_{\mathrm{i}_{\mathrm{rj}}}$, represents $\mathrm{I}$ time task set for the $r_{j}$ type of resource requirements. This paper obtains a total of 10 specific resource requirements at a particular time.

\section{ENERGY CONSUMPTION MODEL}

The energy consumption of the cloud platform for vehicle networking application is mainly divided into 3 parts: the physical server energy consumption, network energy consumption, cooling equipment energy consumption. Among them, the physical server is the key equipment of the cloud platform, so the energy consumption of the physical server is the main part of the cloud platform energy consumption. Therefore, this paper does not consider the network energy consumption and cooling equipment energy consumption.

Energy consumption refers to the energy consumption of a computer system for a period of time, the unit is Joule (J), and its definition is as formula (1):

$$
E=\int_{t}^{t+\Delta t} P d_{t}
$$

Among them, $\mathrm{T}$ stands for the time, $\mathrm{P}$ stands for power consumption, E stands for the total energy consumption of the system. Power consumption is the consumption of energy per unit of time, reflecting the rate of energy consumption of the computer System, the unit is Watt (W).

In this paper, we use the general definition of power consumption in the literature E143, as formula (2):

$$
\mathrm{P}(\mathrm{u}(\mathrm{t}))=\mathrm{kP}_{\max }+(1-\mathrm{k}) \mathrm{P}_{\max } \mathrm{u}(\mathrm{t})
$$

Among them, $p_{\max }$ is the power consumption of the physical server at full load, the article will be set to $250 \mathrm{~V}$. Is 0.6 , the power consumption of the physical server at the time of the load is $60 \%$ of the full load power consumption. This shows that the physical server in the idle state, if it does not shut down or sleep, the power consumption of the physical server is also great. $u_{t}$ for resource utilization, resource utilization rate and the size of the main physical server resource usage is related to the amount of power consumption and CPU resource utilization rate, thus the physical server memory read and write disk read and write throughput, node number and the disk read and write time etc[8].

According to the above description, the energy consumption of the cloud platform for vehicle networking applications $E_{\text {cloudplat }}$ can be seen as the sum of the energy consumption values of all physical servers within the cloud platform, and the definition is as formula (3):

$$
\mathrm{E}_{\text {cloudplat }}=\sum_{\mathrm{i}=1}^{\mathrm{m}} \mathrm{E}_{\mathrm{pm}_{\mathrm{i}}}
$$

$\mathrm{E}_{\mathrm{pm}_{\mathrm{i}}}$ is a physical server power consumption.

From the above analysis, the cloud platform to open the physical server is less, the lower the energy consumption.

\section{ENERGY SAVING INTEGRATION STRATEGY}

For the first problem, algorithm before integration judgment, the resource demand of two tasks or task firstly, integrated set of judges the resources necessary to ensure their needs and less than or equal to the physical resources of the server load, can determine the subsequent integration.

For the second problem, the goal of the algorithm is to integrate different types of tasks in the case of no conflict. In this paper, we refer to the dist measurement standard in the literature [9] to detect the differences between the task sets, and to measure whether the two task sets can be integrated.

Definition: dist is used to describe the differences between the two task sets as formula (4).

$$
\begin{aligned}
& \operatorname{Dist}_{\mathrm{i}, \mathrm{j}}=\sum_{\mathrm{R}_{\mathrm{i}}^{1} \mathrm{R}_{\mathrm{j}}^{2}}\left(\operatorname{aff}_{-} \operatorname{score}\left(\mathrm{R}_{\mathrm{i}}^{1} \mathrm{R}_{\mathrm{j}}^{1}\right) \times \operatorname{Corr}\left(\operatorname{peak}_{\mathrm{i}}^{\mathrm{R}_{1}}, \operatorname{peak}_{\mathrm{j}}^{\mathrm{R}_{1}}\right) \times\right. \\
& \left.\operatorname{Corr}\left(\operatorname{peak}_{\mathrm{i}}^{\mathrm{R}_{2}}, \operatorname{peak}_{\mathrm{j}}^{\mathrm{R}_{2}}\right)\right)
\end{aligned}
$$

$\mathrm{R}_{\mathrm{i}}^{2}, \mathrm{R}_{\mathrm{j}}^{2}$ table from the task set I and or any type of resource, for CPU, memory, disk, network, there are 10 types of combination. Corr is the Pearson correlation coefficient of two task sets for specific resources. In literature [9], it is more necessary to consider the correlation between the peak value and the other values, because the two cooperative tasks are more likely to be in conflict with the peak value. aff_score, a preset value, which ranges 0 to 1,0 said $R_{i}^{2}, R_{j}^{2}$ This two resources in the combination of the minimum conflict; conversely, the maximum of 1 . For example, the two network intensive task set integration will cause large computational overhead, however, if the two task set is directly related to the communication overhead will be greatly reduced, so, for the two network intensive tasks, aff_score is very small.

The meaning of the definition of dist is to integrate the two tasks set with different types of tasks, namely, the resource requirements of the task set is different. But for the same resource requirements of the task set, as long as the same kind of resources in the case of competition can be avoided, but also can be integrated.

Input: list of task set TaskSet, server set Server Output: task set integration scheme Consolidation List 1. initialize the task set list and the server set

2. While TaskSet. size $>=2$ 


\author{
3. Task_i=TaskSet. get $(0)$ \\ 4. for $(\mathrm{j}=1 ; \mathrm{j}<=$ TaskSet. size; $\mathrm{j}++)$ \\ 5. ifTask_i+Task-j<=Server \\ 6. Dist_ij=CaleulateDist(Task_i,Task_j) \\ 7. else \\ 8. icount ++ \\ 9. end if \\ 10. end for \\ 11. if icount $>=$ TaskSet. Size- 1 \\ 12. TaskSet. remove(Task_i) \\ 13. else \\ 14. Integration of Dist_ij and Task_j with minimal Task_i \\ 15. TaskSet. remove(Task_i) \\ 16. TaskSet. remove(Task_j) \\ 17. TaskSet. add(Task_new) \\ 18. endif \\ 19. endfor \\ 20. end while
}

Task set integration algorithm shows how to get the task set integration scheme. The first to reach the set of tasks for information acquisition, remove the task set first task in the list, the rest of the traversal task set in the list of tasks, to find out the integration with the first set of tasks will not exceed the physical server resource load task set, and they were calculated with the first task set dist, dist minimum the task set is selected to be integrated with the first set of tasks, the formation of a new set of tasks after the integration of the update task set list, two before the integration task set delete, add new tasks after the integration of the set.

\section{PERFormance EVAluation}

In the simulation experiment, it is assumed that the cloud platform has enough physical server resources to meet the resource requirements of all the task sets. of tasks:

In this paper, we simulated the task sets with different types

1) Traffic_Analysis, traffic analysis of the collected information for data analysis, can be used to predict traffic conditions, is a computing intensive tasks.

2) File_Zip, file compression, because the data information in the transmission need to be compressed, belonging to the $\mathrm{I} / \mathrm{O}$ intensive and computing intensive tasks.

3) Information_Exchange, the information exchange, the information exchange between the base station and the information processing center, which belong to the communication intensive task.

4) Information_Inquire, information query, including real-time traffic inquiries, near the parking lot, etc., are I / O intensive tasks.

For different types of task sets, there are several aspects of the description:

1) in definition $3\left(\mathrm{vr}_{1}, \mathrm{vr}_{2}, \mathrm{vr}_{3}, \mathrm{vr}_{4}\right)$ to describe the task set for each type of resource demand, this experiment assuming that each server amount of each type is 100 units, "VR" _ i value an integer of 0 to 50 units between. Table I shows the range of resource requirements for different types of tasks.
TABLE I. RANGE OF RESOURCE REQUIREMENTS FOR EACH APPLICATION

\begin{tabular}{lllll}
\hline & vCPU & vMem & vSto & vNet \\
\hline Traffic_Analysis & {$[40,50]$} & {$[30,40]$} & {$[10,20]$} & {$[0,10]$} \\
File_Zip & {$[40,50]$} & {$[10,20]$} & {$[40,50]$} & {$[0,10]$} \\
Information_Exchange & {$[10,20]$} & {$[0,10]$} & {$[30,40]$} & {$[40,50]$} \\
Information_Inquire & {$[10,20]$} & {$[0,10]$} & {$[40,50]$} & {$[30,40]$} \\
\hline
\end{tabular}

2) the expected execution time of each task set is T. In the experiment, the range of $\mathrm{T}$ is an integer between 50 and 100 , and the unit is $\mathrm{S}$.

3) task definition 4 set at a particular time resource demand $\left(\mathrm{T}_{1_{\mathrm{r}_{1}}}, \mathrm{~T}_{2_{\mathrm{r}_{1}}}, \cdots, \mathrm{T}_{10_{\mathrm{r}_{1}}}\right)$ this total get 10 specific time, $\mathrm{T}_{\mathrm{i}_{\mathrm{j}}}$ Is an integer between 1 to 10 .

Figure II for when the number of operations for the 10 time FF algorithm, BF algorithm, TSC algorithm and not to integrate the 4 kinds of physical server active time contrast diagram. We can see from Figure II, when the number of jobs is 10 , without the integration of the case to open a total of 10 physical servers, and 3 kinds of integration algorithms on 3 physical servers, but each physical server of the 3 algorithms of the active time is not the same. In contrast, the BF algorithm is the shortest physical server active time. Although the physical server TSC algorithm in the shortest time is not active, but we can see from Figure III, the energy consumption is the lowest, this is because the physical server energy consumption is not only related to its active time and CPU resource utilization, throughput, memory read and write disk read and write bytes and disk read and write time so, while the TSC algorithm set inaff_score is used to control the corresponding task in integration, to make the integrated energy consumption of a task set is more likely to be integrated.

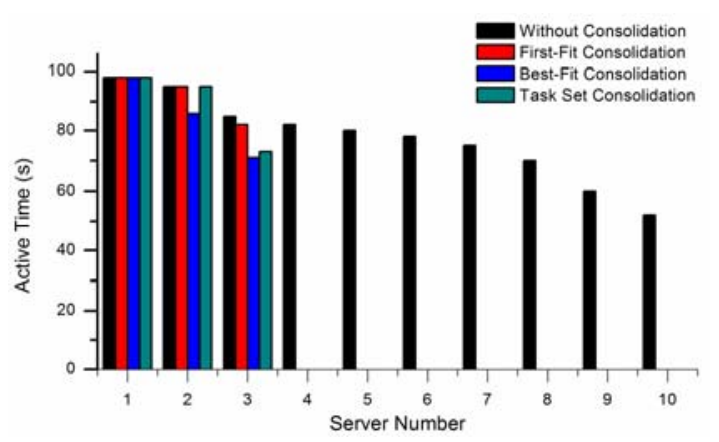

FIGURE I. 10 ACTIVE TIME CONTRAST OF THE PHYSICAL SERVER DURING THE TASK SET

Figure III for the FF algorithm, BF algorithm, TSC algorithm, as well as the integration of the energy consumption of 4 cases of broken line graph. As shown in Figure III, with the increase in the number of tasks for the cloud platform for vehicle networking applications, the energy consumption of these 4 cases is almost linear increase. From the figure it can be seen that, at the beginning of the first task is submitted, except without the integration of the 3 algorithms, the rest of the energy consumption difference; with the increase of time, the amount of submitted tasks increase, differences between the 3 algorithms. Under the same conditions, the TSC algorithm has 
less energy consumption, less than $67.6 \%$ of the energy consumption compared with the non integration. The algorithm reduces the energy consumption by $23.12 \%$ compared with the FF algorithm, and the energy consumption is reduced by $6.48 \%$ compared with the BF algorithm. This shows that the TSC algorithm is the 3 algorithms within such.

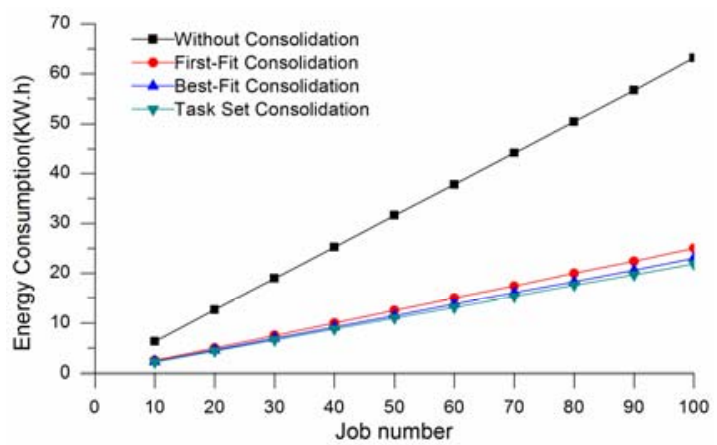

FIGURE II. ENERGY CONSUMPTION LINE CHART

Figure III Comparison of energy consumption for the 4 algorithms. In this paper, the energy consumption of the integrated case is considered as $100 \%$, compared with the other 3 algorithms.

It can be seen from the figure, BF algorithm and TSC algorithm as the number of tasks increases, the energy consumption is more and more obvious advantages, but the FF algorithm because every time is set to integrate the first task list selected randomly from the set of tasks, so the advantage of its energy consumption and not with the number of tasks increases and changes. From Figure IV, we can draw the same conclusion with figure III, that is, the TSC algorithm is the most energy-efficient of the 3 algorithms.

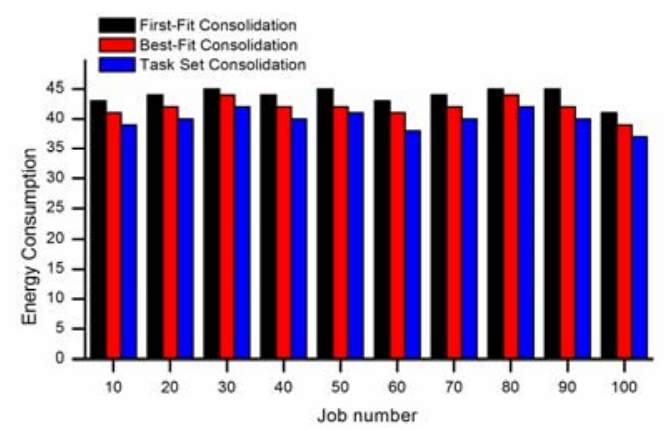

FIGURE III. COMPARISON OF ENERGY CONSUMPTION

Figure $\mathrm{V}$ the number of active servers. As shown in Figure $\mathrm{V}$, the number of active physical servers increases with the number of tasks. As the FF algorithm, BF algorithm and TSC algorithm will have a complete integration of the integration of the task set, so the number of active physical server of the 3 algorithms is the same. Overall, the integration of the task set can greatly reduce the number of physical servers used.

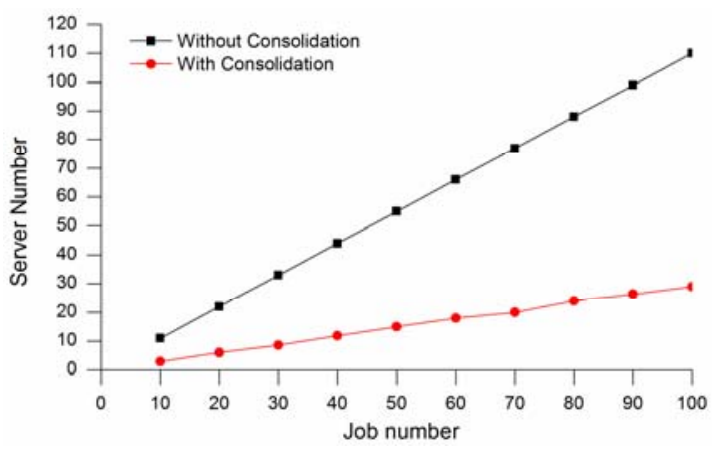

FIGURE IV. THE NUMBER OF ACTIVE WRITING

\section{CONCLUSION}

This paper analyzes the characteristics of execution oriented vehicle networking applications cloud platform, a cloud platform model; analyzes the characteristics of car networking tasks, establish a vehicle networking task set model; analysis of the energy consumption of the system, established the model of energy consumption, combined with the 3 kinds of model provides an energy saving integrated algorithm - task set integration algorithm (TSC). In this paper, the experimental results show that the proposed TSC algorithm can significantly reduce the energy consumption of the network cloud platform, and reduce the number of active physical servers in the premise of ensuring the performance. The next step is to focus on the work of this paper, the task set integration algorithm (TSC) is applied to the practical application of cloud computing platform for vehicle networking.

\section{REFERENCES}

[1] Nelson, Gabe. Connected car's connector: Ethernet[J]. Automotive News,2015,896661:.

[2] [Andrews, Stuart. High-tech health scares[J]. Computer Act!ve,2015,443:

[3] Anonymous. Global light vehicle OE connectivity market- forecasts to 2029: OEM Overview[J]. just - auto,2015,:.

[4] Anonymous. Razer; Razer Wins the CNET 'Best of CES' Award for People's Voice at 2011 International CES[J]. Computer Weekly News, 2011,:

[5] Anonymous. Cie Games announces partnership with Universal Pictures[J]. M2 Presswire,2011,:

[6] Anonymous. Kansas City Honda Dealer Launches Drive Green Car Blog, Video and Social Networking Promotions[J]. Wireless News,2011,:.

[7] Anonymous. SMSC; New Premium SUV Audi Q3 Adopts MOST(R) Technology[J]. Computers, Networks \& Communications,2011,:

[8] Brown, Bob. 7 highlights from Ethernet inventor Bob Metcalfe's Reddit AMA[J]. Network World (Online),2014,:.

[9] Li Wenjun, Zhong Yiming, Li Wenbin, Guo Qing Luo. A Wireless and Real-Time Monitoring System Design for Car Networking Applications[J]. International Journal of Antennas and Propagation,2013: 